\title{
Novel pharmacotherapy: NNI-362, an allosteric p70S6 kinase stimulator, reverses cognitive and neural regenerative deficits in models of aging and disease
}

Nathalie Sumien ${ }^{1}$ D, Matthew S. Wells ${ }^{2,3}$, Akram Sidhu', Jessica M. Wong ${ }^{1}$, Michael J. Forster ${ }^{1}$, Qiao-Xi Zheng ${ }^{2}$ and Judith A. Kelleher-Andersson ${ }^{2^{*}}$

\begin{abstract}
Aging is known to slow the neurogenic capacity of the hippocampus, one of only two mammalian adult neurogenic niches. The reduction of adult-born neurons with age may initiate cognitive decline progression which is exacerbated in chronic neurodegenerative disorders, e.g., Alzheimer's disease (AD). With physiologic neurogenesis diminished, but still viable in aging, non-invasive therapeutic modulation of this neuron regeneration process remains possible. The discovery of truly novel neuron regenerative therapies could be identified through phenotypic screening of small molecules that promote adult-born neurons from human neural progenitor cells (hNPCs). By identifying neuron-generating therapeutics and potentially novel mechanism of actions, therapeutic benefit could be confirmed through in vivo proof-of-concept studies. The key aging and longevity mTOR/p70S6 kinase axis, a commonly targeted pathway, is substrate for potential selective kinase modulators to promote new hippocampal neurons from NPCs. The highly regulated downstream substrate of mTOR, p70S6 kinase, directly controls pleiotropic cellular activities, including translation and cell growth. Stimulating this kinase, selectively in an adult neurogenic niche, should promote NPC proliferation, and cell growth and survival in the hippocampus. Studies of kinase profiling and immunocytochemistry of human progenitor neurogenesis suggest that the novel small molecule NNI-362 stimulates p70S6 kinase phosphorylation, which, in turn, promotes proliferation and differentiation of NPCs to neurons. NNI-362 promoted the associative reversal of age- and disease-related cognitive deficits in aged mice and Down syndrome-modeled mice. This oral, allosteric modulator may ultimately be beneficial for agerelated neurodegenerative disorders involving hippocampal-dependent cognitive impairment, specifically AD, by promoting endogenous hippocampal regeneration.
\end{abstract}

Keywords: Alzheimer's disease, mTOR/p70S6 kinase axis, Allosteric modulator, Human neural progenitors, Hippocampal neuron regeneration, BrdU ${ }^{+}$neuron survival

\footnotetext{
* Correspondence: jkelleher@neuronascent.com

${ }^{2}$ Neuronascent, Inc., 15601 Crabbs Branch Way, Rockville, MD 20855, USA

Full list of author information is available at the end of the article
} 


\section{Introduction}

Neurogenesis in adult humans is an accepted phenomenon following demonstration of $\mathrm{BrdU}^{+}$neurons in the hippocampal dentate gyrus (DG) [1]. However, neurogenesis, in aged individuals and chronic neurodegenerative conditions, remains disputed [2]. An article suggested a lack of measurable human neurogenesis with age [3], while data support continuous neurogenesis throughout aging $[4,5]$ through neurogenic slowing where differentiating neural progenitors may have longer latency phase before becoming functional neurons [6]. Neural stem cell markers appeared age-independent, while proliferative and maturation factors diminished with age, but independently of each other, providing multiple neural targets for intervention [7]. In AD postmortem DG, neurogenesis was reduced and co-neuron maturation factors dropped-off in relation to later Braak stages [8]. The reduced maturation of differentiated early neurons "in waiting" may be causative of age-related dementias [6] and suggests the later stages of neuron maturation are age-critical targets. Antidepressants promoting neuronal progenitor proliferation [9] have minimal benefit on depression in aged patients $[10,11]$, suggesting that enhanced progenitor proliferation is insufficient to promote functioning new neurons. With the continued presence of neural progenitors in aged subgranular zone, this niche remains a putative pharmacotherapeutic target for promoting adult-born neurons.

The standard therapeutic-tested Down syndrome (DS) model, Ts65Dn mice, exhibits many phenotypes of DS and AD [12]. Though Ts65Dn has no direct APP genedosage-linked pathology, other trisomy-related phenotypes provide abundant rationale for testing therapeutics to address memory and neurogenic dysfunction [13]. Reversing the neurogenic deficit in young Ts65Dn mice using fluoxetine provided a memory benefit [14, 15], though the effects' durability in adults remains questionable [16].

New neuron formation in neurodegenerative disorders suggests potential for neuro-compensation as observed in postmortem AD tissue [17], Huntington's patients [18], and stroke in non-human primates [19]. Whether therapies targeting this compensatory neurogenesis are effective in age-related neurodegenerative disorders (ARND) remains undetermined. One non-pharmacotherapy promoting neurogenesis and cognition in aging is aerobic exercise. Human trials demonstrated a 6- or 12-month aerobic fitness program improved cognition, increased hippocampal volume [20, 21], and promoted adult-born neurons to compensate for the loss in the anterior hippocampus [20]. Apparently, the brain retains an innate ability for neuron regeneration even in ARND. Since aging is the greatest risk factor for AD [22], any purported diseasemodifying $\mathrm{AD}$ pharmacotherapy must be effective in compensating for the slowing of adult-born neuron formation and the associated cognitive decline in aging and AD.

The exact [non-therapeutic] trigger that initiates compensatory neurogenesis in ARND is unknown, though growth factors and downstream signaling pathways appear likely candidates. Growth factor mimetics have been tested clinically, exhibiting side effect profiles outweighing any direct gains. However, working downstream at the mTORC1/p70S6 kinase axis would provide a more precise control of protein synthesis and neuron regeneration. Moreover, p70S6 kinase stimulation requires phosphorylation at eight sites, including four auto-inhibitory pseudo-substrate sites [23]. Inhibitors of kinases upstream of p70S6 kinase show benefit as age-related disorder therapeutics, though often associated with toxic side-effects [24]. Without clear/safe targets for promoting adult-born functional neurons, the use of a phenotypic screening platform using hNPCs, and small molecule libraries is a favorable option. The ability to measure neural progenitor proliferation and differentiation to mature neurons in culture has been suggested as necessary to identify novel therapies [25]. Our aim was to discover orally administrated, novel kinase modulators that penetrate the $\mathrm{BBB}$, and promote the formation of functional adult-born neurons from endogenous neural progenitor cells.

\section{Results}

Neuronascent's phenotypic screening platform using hNPCs (Fig. 1a) allowed the discovery of novel neurogenic agents that are also neuroprotective. Due to in silico physicochemical characteristics provided by the commercial library vendor, the number of screened molecules can be narrowed to only BBB-permeable and drug-like compounds. This is critical for small company drug discovery programs in order to quickly progress from the in vitro screening into in vivo efficacy testing. This platform description was expanded from that described by Kelleher-Andersson [25] and revealed a lead candidate: NNI-362 (Fig. 1d). NNI-362 promoted proliferation at $1000 \mathrm{nM}$ versus vehicle $(p<0.05)$ at DIV3 (Fig. 1b) and increased the ratio of mature neurons $\left(\mathrm{MAP}_{2} \mathrm{a}, \mathrm{b}^{+}\right)$to total cells at DIV12/13 at $\geq 1000 \mathrm{nM}$ versus vehicle (all $p<0.05$ ) (Fig. 1c).

Young mice spent more time with the novel object than old mice $(p<0.05)$ and old NNI-362-treated mice exhibited a similar pattern to the young mice $(p<0.05$ vs. old controls) (Fig. 2a). Old mice had lower new $\mathrm{BrdU}^{+}$cells $(p<0.05)$, while the old $10 \mathrm{mg} / \mathrm{kg}$ NNI-362 dose had higher $\mathrm{BrdU}^{+}$cells migrating/surviving than the old controls $(p<0.05)$ and were not significantly different from the young $(p=0.08)$ (Fig. 2b). This effect was associated with an improvement in memory 


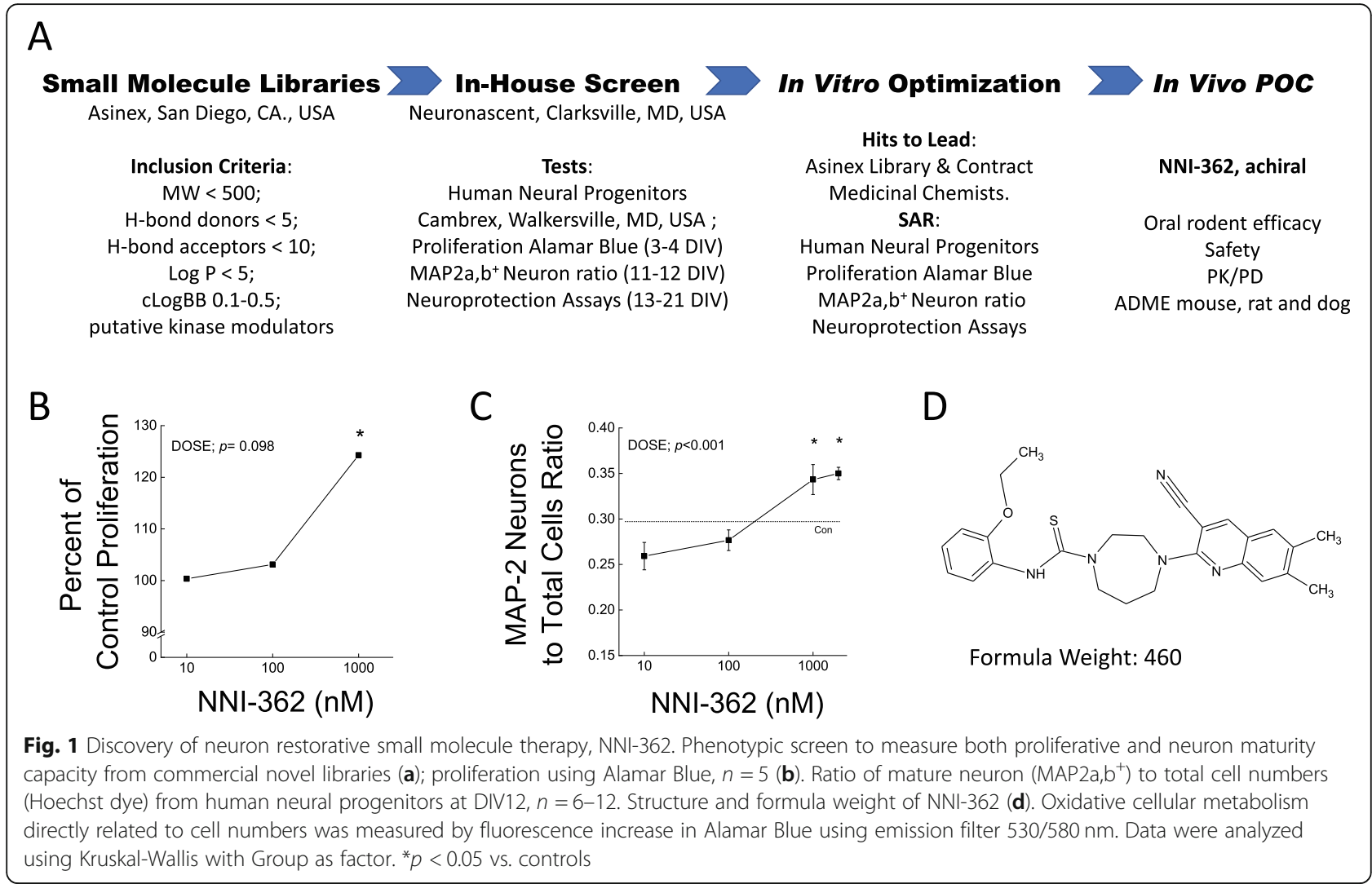

function only at the $10 \mathrm{mg} / \mathrm{kg}$ dose (data not shown) similar to Fig. 2a. NNI-362 did not reverse age-related motor dysfunction (Fig. 2c). Similarly, in Ts65Dn mice, NNI-362 treatment was associated with reversal of DSmodeled memory impairment $(p<0.05)$ (Fig. $2 \mathrm{~d}$ ) and decreased number of mature $\mathrm{BrdU}^{+}$cells surviving in the DG $(p<0.05)$ (Fig. 2e). NNI-362 did not reduce the hyperactivity observed in Ts65Dn mice (Fig. 2f). NNI351 was also discovered through the phenotypic screen (Fig. 1a); however, it lacked the neuroprotection capacity in vitro and did not improve memory during aging or consistently in the DS model (Fig. 2), yet did reduce hyperactivity (Fig. 2f).

NNI-362 only significantly stimulated the p70S6 kinase from the 151 kinase panel (Fig. 3a). No competition at the active site of p70S6 nor any of the other pathwayrelated kinases were observed, providing evidence for allosteric targeting by NNI-362 (Fig. 3b). Only at the neuron regenerative concentration $(\geq 1000 \mathrm{nM})$ and only during the early dividing and beginning differentiation phase was p70S6 kinase selectively phosphorylated (6DIV) significantly $(p<0.05)$, while being absent in fully differentiated neurons at 12DIV (Fig. 3c, d). The switching on of translation and increased neurogenesis is possible through numerous targeting points of the mTOR/
p70S6 kinase axis [23], but selectivity to neural cells may be only possible by means of predominantly neuronal CDK5 kinase phosphorylation at p70S6 kinase autoinhibitory site [26]. Results confirm only the addition of CDK5 inhibitors, indirubin and BML259, ameliorated NNI-362-induced proliferation, while the addition of other inhibitors, including p38 kinase inhibitor, was ineffective (Fig. 3e, f). These results suggest that NNI-362 acts at the auto-inhibitory pseudo-substrate site, Ser411, where CDK5 phosphorylates p70S6 kinase (Fig. 3g) during the mitogenic translational stage, not the terminally differentiated neuron stage.

\section{Discussion}

The main findings from this study were that NNI-362 promoted proliferation and survival of adult-born neural cells associated with reversal of cognitive deficits, without toxic or off-target effects up to 6 weeks of administration, and acts allosterically and downstream of mTOR.

The screening platform allowed for the discovery of small molecule therapies that promote proliferation and increase new mature neuron numbers in culture, through unique kinase modulating pathway(s). This phenotype is obligatory for the compound to succeed as 


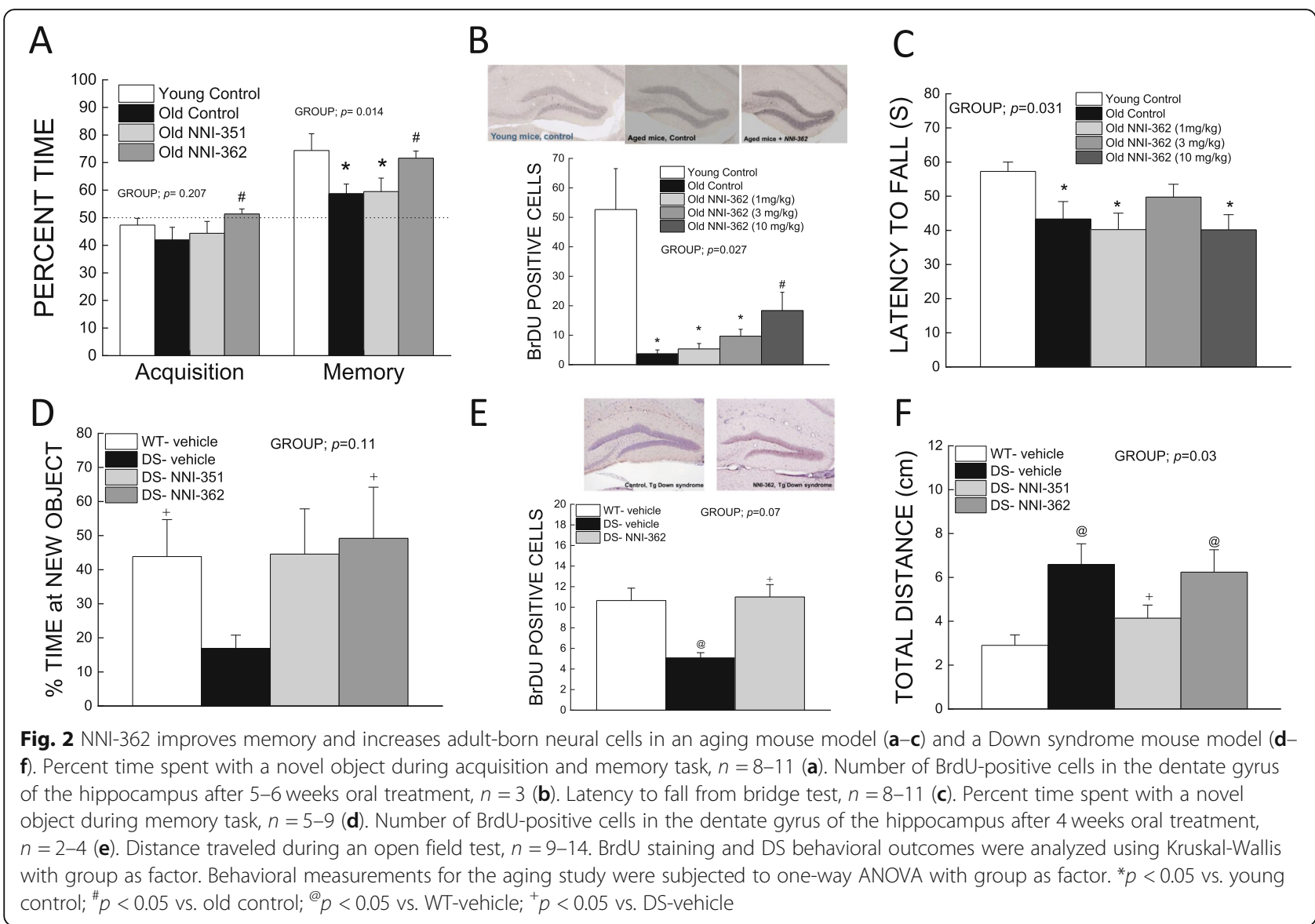

a human therapy for age-related degenerative disorders. A therapeutic candidate that is only neural progenitorproliferative appears unable to reverse age-related cognitive impairment [11]. Fluoxetine had neurogenic and behavioral benefit in young TS65Dn mice $[14,15]$, but not in adult mice [16]. Noteworthy, NNI-351, a compound with only a proliferative phenotype $\left(\mathrm{Ki} 67^{+}\right.$cells greater than DS-vehicle, data not shown), reduced hyperactivity, but was ineffective in reversing memory deficits in aging and DS models, suggesting that maturation and survival of adult-born neural cells are necessary for cognitive improvements in aging and neurodegenerative disease. Indeed, brain aging may be more complex especially in the adult hippocampal neurogenic niche, where "stem cell exhaustion" [27] may be less critical than promotion of maturation of dividing neural progenitors/early neurons to fully mature, functioning neurons. Boldrini et al. [4] suggest the human neural progenitor hippocampal pool remains available for neurogenesis into our 80s. This need for promotion of maturation of the adult-born neurons appeared more critical in AD than aging alone [8]. Further studies of differentiation and synaptic integration will be needed to conclusively resolve that NNI-
362 has an effect on neurogenesis beyond proliferation, migration, and adult-born cell survival.

Identifying the mechanism of action of NNI-362 by which neural cells proliferate, migrate, survive, and improve cognitive function could lead to novel target identification for disease-modifying neuron regeneration. While a selective p70S6 kinase inhibitor is known [28], our data indicate that NNI-362 stimulates p70S6 kinase, suggesting activity at a critical auto-inhibitory allosteric site: Ser411 (conserved across many species [29]), a substrate for CDK5/activator phosphorylation, suggesting selectivity to neural tissue [30]. Inhibition of CDK5 ameliorated NNI-362-induced proliferation accounting for NNI-362's neuron selectivity, while other upstream kinases or their inhibitors played no definitive role in induced neuron generation.

\section{Conclusion}

Elucidating the CDK5/activator substrate Ser411 site on p70S6 kinase [26] that is selectively stimulated in neural cells by NNI-362 provides greater insights into how this clinical-stage therapeutic works in aging brain and neurodegenerative disorders. Further studies will look at 


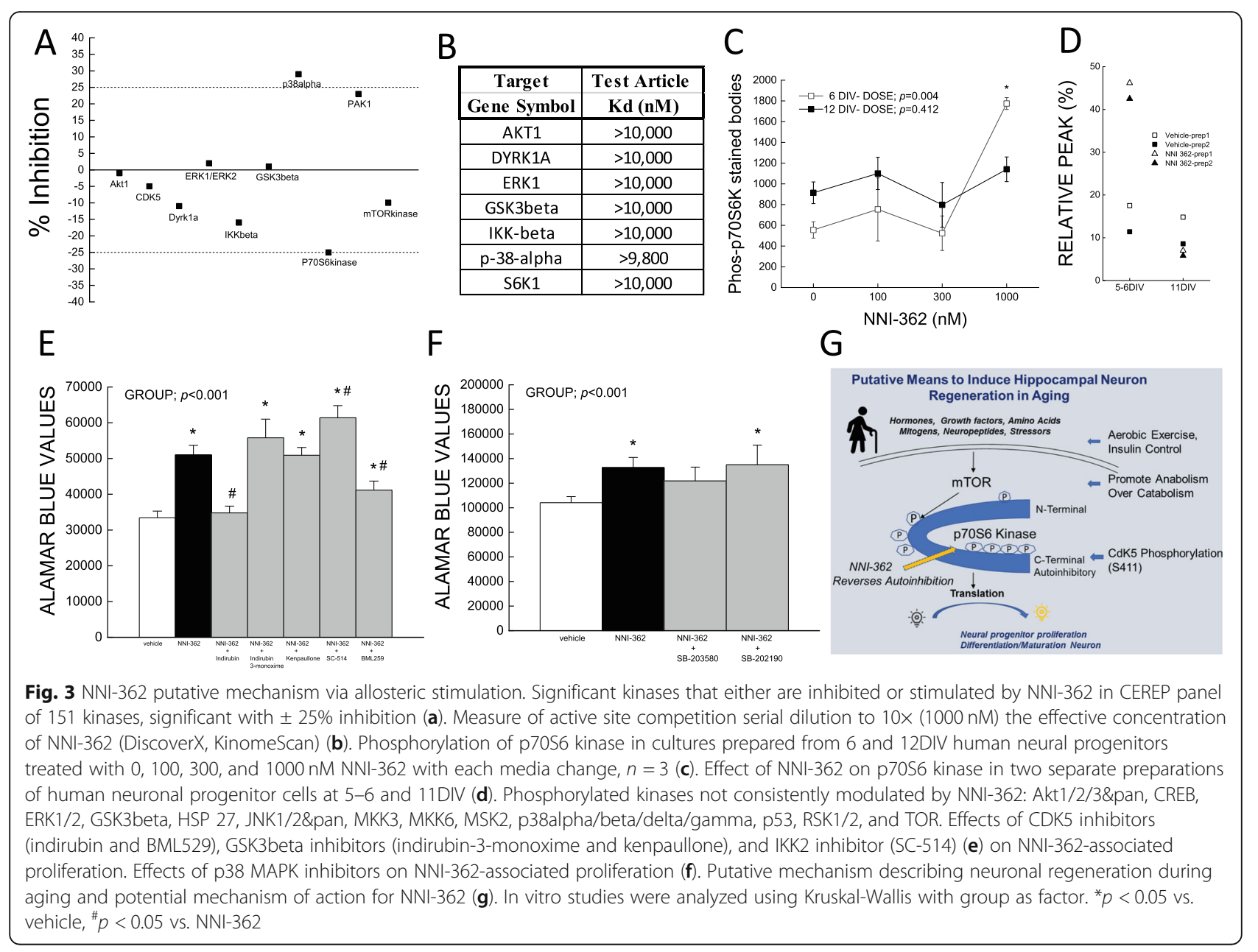

selective neuron regeneration markers and reversal of cognitive deficits in aging and neurodegenerative models and the level of allosteric site stimulation on p70S6 kinase by Western and MAP kinase profiling in vivo. Though mTOR inhibition has been suggested to promote longevity, our data support a downstream target to improving brain "healthspan": restorative capacity via neural-selective allosteric stimulation of p70S6 kinase.

\section{Supplementary Information}

The online version contains supplementary material available at https://doi. org/10.1186/s13287-020-02126-3.

\section{Additional file 1.}

\section{Acknowledgements}

N/A

\section{Authors' contributions}

Therapy invention: JAK; concept and experimental design: JAK, NS, and MJF; experimental procedures: MW, Q-XZ, JMW, and AS. Analyses and writing: JAK and NS. The authors read and approved the final manuscript.

\section{Funding}

This work was supported by Neuronascent, Inc., and the National Institutes of Health [2P01 AG022550 (PI-NS)]. NIA has supported the development of NNI-362 through a translational safety program (Dr. Petanceska-directed program) and through the IND/phase 1a clinical trial (R01 AG056561, PI-JAK). $\mathrm{NNN}-362$ is presently in phase 1a clinical trial for Alzheimer's disease NCT04074837.

Availability of data and materials

The datasets used and/or analyzed during the current study are available from the corresponding author on reasonable request.

Ethics approval and consent to participate N/A.

\section{Consent for publication}

N/A.

\section{Competing interests}

JAK is a majority stockholder in Neuronascent. No other conflict exists.

\section{Author details}

'Department of Pharmacology \& Neuroscience, University of North Texas Health Science Center, 3500 Camp Bowie Blvd., Fort Worth, TX, USA.

${ }^{2}$ Neuronascent, Inc., 15601 Crabbs Branch Way, Rockville, MD 20855, USA.

${ }^{3}$ Novavax, 21 Firstfield Rd., Gaithersburg, MD 20878, USA. 
Received: 6 August 2020 Accepted: 27 December 2020

Published online: 13 January 2021

\section{References}

1. Eriksson PS, et al. Neurogenesis in the adult human hippocampus. Nat Med. 1998:4(11):1313-7.

2. Kumar A, et al. Adult neurogenesis in humans: a review of basic concepts, history, current research, and clinical implications. Innov Clin Neurosci. 2019; 16(5-6):30-7.

3. Sorrells SF, et al. Human hippocampal neurogenesis drops sharply in children to undetectable levels in adults. Nature. 2018;555(7696):377-81.

4. Boldrini $M$, et al. Human hippocampal neurogenesis persists throughout aging. Cell Stem Cell. 2018;22(4):589-99 e5.

5. Spalding $\mathrm{KL}$, et al. Dynamics of hippocampal neurogenesis in adult humans. Cell. 2013;153(6):1219-27.

6. Kempermann $\mathrm{G}$, et al. Human adult neurogenesis: evidence and remaining questions. Cell Stem Cell. 2018;23(1):25-30.

7. Mathews KJ, et al. Evidence for reduced neurogenesis in the aging human hippocampus despite stable stem cell markers. Aging Cell. 2017;16(5):11959.

8. Moreno-Jimenez EP, et al. Adult hippocampal neurogenesis is abundant in neurologically healthy subjects and drops sharply in patients with Alzheimer's disease. Nat Med. 2019;25(4):554-60.

9. Santarelli $L$, et al. Requirement of hippocampal neurogenesis for the behavioral effects of antidepressants. Science. 2003;301(5634):805-9.

10. Cowen DS, et al. Age-dependent decline in hippocampal neurogenesis is not altered by chronic treatment with fluoxetine. Brain Res. 2008;1228:14-9.

11. Couillard-Despres $\mathrm{S}$, et al. Ageing abolishes the effects of fluoxetine on neurogenesis. Mol Psychiatry. 2009;14(9):856-64.

12. Gardiner KJ. Pharmacological approaches to improving cognitive function in Down syndrome: current status and considerations. Drug Des Devel Ther. 2015;9:103-25.

13. Hamlett ED, et al. Cognitive impairment, neuroimaging, and Alzheimer neuropathology in mouse models of Down syndrome. Curr Alzheimer Res. 2016;13(1):35-52.

14. Bianchi $P$, et al. Early pharmacotherapy restores neurogenesis and cognitive performance in the Ts65Dn mouse model for Down syndrome. J Neurosci. 2010;30(26):8769-79.

15. Clark S, et al. Fluoxetine rescues deficient neurogenesis in hippocampus of the Ts65Dn mouse model for Down syndrome. Exp Neurol. 2006;200(1): 256-61.

16. Heinen $\mathrm{M}$, et al. Adult-onset fluoxetine treatment does not improve behavioral impairments and may have adverse effects on the Ts65Dn mouse model of Down syndrome. Neural Plast. 2012;2012:467251.

17. Jin K, et al. Increased hippocampal neurogenesis in Alzheimer's disease. Proc Natl Acad Sci U S A. 2004;101(1):343-7.

18. Curtis MA, et al. Increased cell proliferation and neurogenesis in the adult human Huntington's disease brain. Proc Natl Acad Sci U S A. 2003;100(15): 9023-7

19. Koketsu D, et al. Increased number of new neurons in the olfactory bulb and hippocampus of adult non-human primates after focal ischemia. Exp Neurol. 2006:199(1):92-102

20. Erickson Kl, et al. Exercise training increases size of hippocampus and improves memory. Proc Natl Acad Sci U S A. 2011;108(7):3017-22.

21. Jonasson LS, et al. Aerobic exercise intervention, cognitive performance, and brain structure: results from the physical influences on brain in aging (PHIBRA) study. Front Aging Neurosci. 2016;8:336.

22. 2020 Alzheimer's disease facts and figures. 2020. Alzheimers Dement., 2020.

23. Magnuson B, Ekim B, Fingar DC. Regulation and function of ribosomal protein S6 kinase (S6K) within mTOR signalling networks. Biochem J. 2012; 441(1):1-21

24. Hu S, et al. GSK3 inhibitors show benefits in an Alzheimer's disease (AD) model of neurodegeneration but adverse effects in control animals. Neurobiol Dis. 2009;33(2):193-206.

25. Kelleher-Andersson J. Discovery of neurogenic, Alzheimer's disease therapeutics. Curr Alzheimer Res. 2006;3(1):55-62

26. Zhou Y, et al. Increased phosphorylation of p70 S6 kinase is associated with HPV16 infection in cervical cancer and esophageal cancer. Br J Cancer. 2007; 97(2):218-22.

27. Lopez-Otin C, et al. The hallmarks of aging. Cell. 2013;153(6):1194-217.
28. Pearce LR, et al. Characterization of PF-4708671, a novel and highly specific inhibitor of p70 ribosomal S6 kinase (S6K1). Biochem J. 2010;431(2):245-55.

29. Hou Z, He L, Qi RZ. Regulation of $s 6$ kinase 1 activation by phosphorylation at ser-411. J Biol Chem. 2007;282(10):6922-8.

30. Dhavan R, Tsai LH. A decade of CDK5. Nat Rev Mol Cell Biol. 2001;2(10): 749-59.

\section{Publisher's Note}

Springer Nature remains neutral with regard to jurisdictional claims in published maps and institutional affiliations.
Ready to submit your research? Choose BMC and benefit from:

- fast, convenient online submission

- thorough peer review by experienced researchers in your field

- rapid publication on acceptance

- support for research data, including large and complex data types

- gold Open Access which fosters wider collaboration and increased citations

- maximum visibility for your research: over $100 \mathrm{M}$ website views per year

At BMC, research is always in progress.

Learn more biomedcentral.com/submissions 